$\frac{40}{81} 15.95$ gs (2)

PREPARED FOR THE U.S. DEPARTMENT OF ENERGY, UNDER CONTRACT DE-AC02-76-CHO-3073

PPPL-3114

UC-420

PPPL-3114

\title{
ALFVÉN CYCLOTRON INSTABILITY AND ION CYCLOTRON EMISSION
}

BY

N.N. GORELENKOV AND C.Z. CHENG

JULY 1995
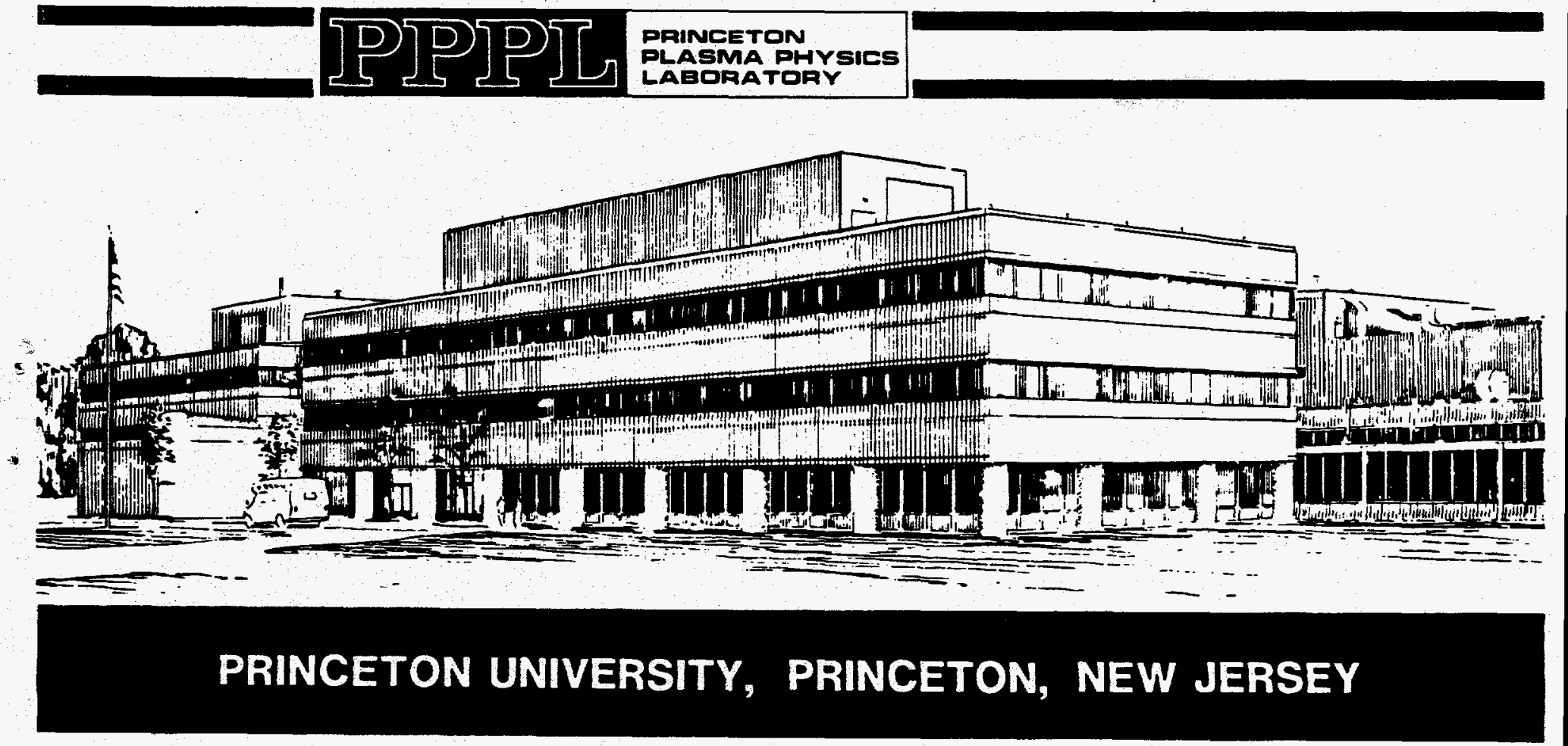


\section{NOTICE}

This report was prepared as an account of work sponsored by an agency of the United States Government. Neither the United States Government nor any agency thereof, nor any of their employees, makes any warranty, express or implied, or assumes any legal liability or responsibility for the accuracy, completeness, or usefulness of any information, apparatus, product, or process disclosed, or represents that its use would not infringe privately owned rights. Reference herein to any specific commercial produce, process, or senvice by trade name, trademark, manufacturer, or otherwise, does not necessarily constitute or imply its endorsement, recommendation, or favoring by the United States Government or any agency thereof. The views and opinions of authors expressed herein do not necessarily state or reflect those of the United States Government or any agency thereof.

\section{NOTICE}

This report has been reproduced from the best available copy. Available in paper copy and microfiche.

Number of pages in this report: 26

DOE and DOE contractors can obtain copies of this report from:

Office of Scientific and Technical Information

P.O. Box 62

Oak Ridge, TN 37831 ;

(615) $576-8401$.

This report is publicly available from the:

National Technical Information Service

Department of Commerce

5285 Port Royal Road

Springfield, Virginia 22161

(703) $487-4650$ 


\section{DISCLAIMER}

Portions of this document may be illegible in electronic image products. Images are produced from the best available original document. 


\title{
Alfvén Cyclotron Instability and Ion Cyclotron Emission
}

\author{
N. N. GORELENKOV*and C. Z. CHENG \\ Princeton Plasma Physics Laboratory, Princeton University, \\ P. O. Box 451, Princeton, New Jersey 08543 USA
}

\begin{abstract}
Two-dimensional solutions of compressional Alfvén eigenmodes (CAE) are studied in the cold plasma approximation. For finite inverse aspect ratio tokamak plasmas the two-dimensional eigenmode envelope is localized at the low magnetic field side with the radial and poloidal localization on the order of $a / \sqrt{m}$ and $a / \sqrt[4]{m}$, respectively, where $m$ is the dominant poloidal mode number. Charged fusion product driven Alfvén Cyclotron Instability (ACI) of the compressional Alfvén eigenmodes provides the explanation for the ion cyclotron emission (ICE) spectrum observed in tokamak experiments. The ACI is excited by fast charged fusion products via Doppler shifted cyclotron wave-particle resonances. The ion cyclotron and electron Landau dampings and fast particle instability drive are calculated perturbatively for deuterium-deuterium (DD) and deuterium-tritium (DT) plasmas. Near the plasma edge at the low field side the velocity distribution function of charged fusion products is localized in both pitch angle and velocity. The poloidal localization of the eigenmode enhances the ACI growth rates by a factor of $\sqrt{m}$ in comparison with the previous results [N. N. Gorelenkov and C. Z. Cheng, to appear in Phys. Plasmas, June (1995)] without poloidal envelope. The thermal ion cyclotron damping determines that only modes with eigenfrequencies at multiples of the edge cyclotron frequency of the background ions can be easily excited and form an ICE spectrum similar to the experimental observations. Theoretical understanding is given for the results of TFTR DD and DT experiments with $v_{\alpha 0} / v_{A}<1$ and JET experiments with $v_{\alpha 0} / v_{A}>1$.
\end{abstract}

PACS numbers: $52.35 . \mathrm{Qz}, 52.55 . \mathrm{Fa}$

${ }^{*}$ Permanent address: Troitsk Institute for Innovative and Fusion Research, Troitsk, Moscow region, Russia, 142092 


\section{INTRODUCTION}

The Ion Cyclotron Emission (ICE) in hot and dense plasmas has been studied in experiments on Joint European Torus (JET) [1, 2] and Tokamak Fusion Test Reactor (TFTR) [3, 4] (see also references contained therein). The frequency spectra of ICE signal present sharp peaks at harmonics of bulk ion cyclotron frequency evaluated at the low magnetic field side of the plasma edge. Typically, the ICE spectra show a broadband part for the frequency $\omega>5 \omega_{c D e d g e}$ and regular spaced peaks with $\omega=l \omega_{c D e d g e}$, where $\omega_{c D e d g e}$ is the outer edge deuterium cyclotron frequency. The narrow width of ICE spectrum peaks $\left(\Delta \omega / \omega \ll a / R_{0}\right.$, where $a$ and $R_{0}$ are the minor and major radii of the tokamak plasma, respectively) indicates that the ICE is localized in the low field side of the tokamak. The correlation between the amplitude of ICE spectral peaks and the neutron emission rate was observed in JET [1] and in TFTR [4] in deuterium-deuterium (DD) and deuterium-tritium (DT) experiments. The experimental results, reviewed in Ref. [1] clearly demonstrate that charged fusion products provide the free energy to generate ICE.

However, ICE in TFTR [4] has special features different form the JET observations. At the beginning of NBI, peaks of the ICE signal correspond to the harmonics of $H e^{3}$ and $T$ charged products. Later on, the spectra peaks are determined by thermal ion damping in DD, DT and TT discharges. The broadband part of the ICE signal does not correlate with the neutron emission rate.

In this paper the ICE is considered to be the resonant cyclotron instability of compressional Alfvén (fast Alfvén, or magnetosonic) eigenmodes (CAE) driven by fast fusion product ions. Similar approach has been used in earlier studies [5-7], where the excitation of Alfvén Cyclotron Instability (ACI) is associated with the free energy source of fast charged fusion product ions which are in cyclotron resonance with compressional Alfvén waves. However, in these papers [5-7] the ICE spectra were explained only in terms of the excitation mechanism. But, in the paper by N. N. Gorelenkov and C. Z. Cheng [8] it was pointed out qualitatively that the thermal ion cyclotron damping plays an essential role in forming the ACI (therefor ICE) spectra.

In papers $[5,6] \mathrm{ACI}$ has been studied in the uniform plasma approximation by neglecting

the fast particle drift motion. The instability growth rate is proportional to $\sqrt{n_{\alpha} / n_{e}}$, where $n_{\alpha}$ and $n_{e}$ are the densities of fast particles and electrons, respectively.

The extension of the uniform plasma theory to include inhomogeneous magnetic field was reviewed by A. B. Mikhailovskii [9]. The contribution to the plasma permeability tensor from fast particles was calculated by including the particle drift motion and bounce resonances. The fast particle radial orbit width was assumed negligible in comparison with the radial wavelength. It was shown that the instability with growth rate proportional to $\sqrt{n_{\alpha} / n_{e}}$ is stabilized in the tokamak plasma with an inhomogeneous magnetic field if the wave propagates perpendicularly to the magnetic field.

In paper [8] the nonlocal contribution to the permeability tensor was calculated in the drift approximation for fast particles by keeping only the resonance part of the tensor (see also Ref. [10]). The CAE - fast particle interaction is determined by the cyclotron resonance inside the layer 
which was assumed small in comparison to the mode envelope and the plasma parameter scale lengths. The fast particle resonance contribution to the plasma dispersion leads to ACI with growth rates proportional to $n_{\alpha} / n_{e}$. Typically, these growth rates are small in tokamak plasmas. It implies that in inhomogeneous plasma one should look for an absolute instability with localized origin rather than a convective type instability, which convects the wave packet away before the wave amplitude becomes large. Since the ACI growth rates are proportional to $n_{\alpha} / n_{e}$, one expects that in the nonlinear regime the amplitude of ICE signal will be proportional to $n_{\alpha} / n_{e}$ and the neutron emission rate.

In papers $[7,11]$ the two-dimensional fast wave eigenmode structure was obtained in the large aspect ratio limit $\left(\epsilon=a / R_{0} \ll 1\right)$ without the poloidal envelope structure. A local analysis of the wave-particle interaction was performed.

In this paper, the two-dimensional CAE structure will be presented in Section 2 for the cold tokamak plasma with finite inverse aspect ratio. Based on the two-dimensional eigenmode structure the thermal ion cyclotron and electron Landau damping rates are calculated perturbatively in Sec. 3. It is shown that only modes with eigenfrequencies at multiples of the edge cyclotron frequency of background ions can be easily excited and can form the ICE spectrum similar to the experimentally observed. In Sec. 4 we employ the formalism developed in Ref.[8] to calculate perturbatively the ACI growth rates driven by both fusion protons and $\alpha$-particles in deuterium-tritium (DT) tokamak plasmas. The fast particle distribution function is assumed to be localized in both pitch angle and velocity near the plasma edge. The conclusions are given in Sec. 5 .

\section{COMPRESSIONAL ALFVÉN EIGENMODES}

\subsection{Eigenmode Equation}

In the range of ion cyclotron frequency compressional Alfvén waves have been considered to be responsible for the ICE. To obtain the equation for localized compressional Alfvén eigenmodes (CAE) we consider the model of inhomogeneous, magnetized plasma in a tokamak with a circular cross section. By neglecting the background ion and electron kinetic effects and with vanishing parallel electric field, the perturbed electric field satisfies Faraday's and Amper's laws which reduce to the following equation:

$$
\nabla \times \nabla \times \mathbf{E}=\frac{\omega^{2}}{c^{2}} \hat{\boldsymbol{\epsilon}} \cdot \mathbf{E},
$$

where we assume that $\mathbf{E}$ has two components perpendicular to the equilibrium magnetic field and $\mathbf{E}(t) \sim \exp (-i \omega t)$. The cold plasma permeability tensor $\hat{\boldsymbol{\epsilon}}$ has elements

$$
\hat{\epsilon}_{11}=\hat{\epsilon}_{22}=\sum_{i} \frac{\omega_{p i}^{2}}{\omega_{c i}^{2}-\omega^{2}},
$$




$$
\hat{\epsilon}_{12}=-\hat{\epsilon}_{21}=i \sum_{i} \frac{\omega}{\omega_{c i}} \frac{\omega_{p i}^{2}}{\omega_{c i}^{2}-\omega^{2}}
$$

for frequency $\omega$ lower than low hybrid frequency, where $\omega_{c i}$ and $\omega_{p i}$ are the ion cyclotron and ion plasma frequencies, respectively. Note that the tensor Eq. (3) is rotationally invariant, which means that it can be used for any orthogonal coordinates perpendicular to the equilibrium magnetic field $\mathbf{B}$. Introducing parallel component of wave vector as $k_{\|}=-i \mathbf{b} \cdot \nabla \ln E$ and choosing direction 1 as the radial direction and direction 2 as the poloidal direction, we can rewrite Eq. (1) as a system of two coupled equations for the radial and poloidal components of the perturbed electric field

$$
\begin{aligned}
& \left(-\frac{1}{r^{2}} \frac{\partial^{2}}{\partial \theta^{2}}-F\right) E_{r}+\left(\frac{\partial}{\partial \theta} \frac{1}{r^{2}} \frac{\partial}{\partial r} r-H\right) E_{\theta}=0 \\
& \left(\frac{\partial}{\partial r} \frac{1}{r} \frac{\partial}{\partial \theta}+H\right) E_{r}+\left(-\frac{\partial}{\partial r} \frac{1}{r} \frac{\partial}{\partial r} r-F\right) E_{\theta}=0
\end{aligned}
$$

where $F=\frac{\omega^{2}}{c^{2}} \hat{\epsilon}_{11}-k_{\|}^{2}$ and $H=\frac{\omega^{2}}{c^{2}} \hat{\epsilon}_{12}$. To further simplify these equations we make use of the assumptions: $\partial \ln E / \partial \theta \gg \partial \ln E / \partial r \gg 1, \partial \ln \left(\hat{\epsilon}_{12}, \hat{\epsilon}_{11}\right) / \partial(r, \theta) \ll \partial \ln E / \partial(r, \theta)$, and $\partial \hat{\epsilon}_{i j} / \partial \theta \ll$ $\partial \hat{\epsilon}_{i j} / \partial r$. The first assumption implies radially localized solutions [7, 8, 11]. The system of equations (4) can be simplified by multiplying the first equation with $\left(r^{-2}(\partial / \partial r) r(\partial / \partial \theta)+H\right)$, the second equation with $\left(r^{-2}\left(\partial^{2} / \partial \theta^{2}\right)+F\right)$, and summing them. tdt2432926 Finally, we obtain the eigenmode equation

$$
\left[\frac{1}{r} \frac{\partial}{\partial r} r \frac{\partial}{\partial r}+\frac{1}{r^{2}} \frac{\partial^{2}}{\partial \theta^{2}}+\frac{F^{2}+H^{2}}{F}+\frac{1}{r F}\left(\frac{\partial}{\partial r} H\right) \frac{\partial}{\partial \theta}\right] E_{\theta}=0
$$

which is Eq. (39) of Ref. [11]. Analytical solutions of Eq. (5) can be obtained in the limit $\omega^{2} \gg \omega_{c i}^{2}$. In Sec. 3 we will use the analytical solution for qualitative analysis of CAE damping at $\omega \sim \omega_{c i}$. In the limits of $\omega^{2} \gg \omega_{c i}^{2} a n d k_{\|}^{2} \ll m^{2} / r^{2}$, Eq. (5) is reduced to

$$
\left[\frac{1}{r} \frac{\partial}{\partial r} r \frac{\partial}{\partial r}+\frac{1}{r^{2}} \frac{\partial^{2}}{\partial \theta^{2}}+\frac{\omega^{2}}{v_{A}^{2}}\right] E_{\theta}=0
$$

where $v_{A}^{2}=c^{2} \bar{\omega}_{c} \omega_{c e} / \omega_{p e}^{2}=\left(B^{2} / 4 \pi n_{e}\right) \sum_{i=1,2}\left(z_{i}^{2} n_{i} / m_{i} n_{e}\right)$ is the Alfvén velocity for a two-component plasma, and $z_{i}$ and $n_{i}$ are the electric charge and density of ion species $i$, respectively. Equation (6) has been solved in the cylindrical plasma approximation $[11,13]$ and in the toroidal geometry $[8,11]$ using small parameters $1 / m$ and $\epsilon$, where $m \simeq \partial \ln E / \partial \theta$ is the poloidal mode number.

\subsection{Cylindrical Eigenmode Solution}

For simplicity we choose the plasma density profile in the form

$$
n(r)=n_{0}\left(1-r^{2} / a^{2}\right)^{\sigma_{i}},
$$


where $r$ is the minor radius, and $n_{0}$ is the ion plasma density at the magnetic axis. Without the toroidal coupling the cylindrical solution has the form $E_{\theta}=\hat{E}(r) \exp [-i \omega t-i n \varphi+i m \theta]$, where $\theta$ is the poloidal angle, $\varphi=z / R_{0}, R_{0}$ is the major radius of the magnetic axis and $z$ is the coordinate along the plasma cylindrical axis. The eigenmode radial structure is described by the following equation:

$$
\left(\frac{1}{r} \frac{\partial}{\partial r} r \frac{\partial}{\partial r}-V(r)\right) \hat{E}(r)=0
$$

where the potential is given by

$$
V(r) \simeq \frac{m^{2}}{r^{2}}-\frac{\omega^{2}}{v_{A}^{2}(0)} \frac{n(r)}{n_{0}}
$$

$v_{A}^{2}(0)$ is evaluated at the magnetic axis with the vacuum toroidal magnetic field. If $V(r)$ forms a potential well, Eq. (8) can be approximated as a harmonic oscillator equation which admits localized solutions. For chosen plasma profile Eq. (7) and $m \gg 1$, a potential well is formed at $r=r_{0}$, where $r_{0}^{2} / a^{2}=1 /\left(1+\sigma_{i}\right)-(2 s+1) \Delta^{2} / a^{2}, \Delta^{2} / a^{2}=\sqrt{2 \sigma_{i} /\left(1+\sigma_{i}\right)} /\left[m\left(1+\sigma_{i}\right)\right]$, and $s$ is an integer and represents the radial wavenumber. The localized eigenmode solutions have the eigenfrequency $[7,13]$

$$
\omega^{2}=\frac{m^{2} v_{A}^{2}\left(r_{0}\right)}{r_{0}^{2}}\left(1+\frac{\left(1+\sigma_{i}\right)}{\sigma_{i}} \frac{(2 s+1) \Delta^{2}}{r_{0}^{2}}\right)
$$

and the eigenfunction

$$
\hat{E}(r)=\hat{E}_{0} \phi_{s}\left(\frac{\sqrt{2}\left(r-r_{0}\right)}{\Delta}\right)
$$

where $\phi_{s}(x)=e^{-x^{2} / 2} H_{s}(x) / \sqrt{2 ! 2^{s} \sqrt{2}}$ and $H_{s}$ are the $s-t h$ order Chebyshev-Hermit functions and polynomials, respectively. Note that in the potential well region the solution (11) is consistent with the ordering

$$
\frac{\partial E_{\theta}}{\partial r} \sim \frac{\sqrt{m} E_{\theta}}{r}, V(r) \sim \frac{m}{r^{2}} .
$$

For $\sigma_{i} \leq 1$ the eigenmode is localized near the plasma edge. Thus, to form a potential well, a rapid variation of the plasma density profile is required near the plasma edge.

\subsection{Toroidal Eigenmode Solution}

In Eq. (6) toroidal effects are included in terms of the poloidal dependence of the equilibrium magnetic field $B=B_{0} /(1+\epsilon \cos \theta)$, where $\epsilon=r / R_{0}, B_{0}$ is the magnetic field at the magnetic 
axis. We will consider modes which satisfy the cylindrical orderings, Eq. (12). Then, in the lowest order in $(1 / m)$ Eq. (6) reduces to

$$
\frac{1}{r^{2}} \frac{\partial^{2} E_{\theta}}{\partial \theta^{2}}=-\frac{\omega^{2}}{v_{A}^{2}(0)} \frac{n(r)}{n_{0}}(1+\epsilon \cos \theta)^{2} E_{\theta} .
$$

This equation has a solution to the lowest order in the form [11]

$$
E_{\theta}=\hat{E}(r, \theta) \exp \left[-i \omega t+i m\left(\theta+\epsilon_{0} \sin \theta\right)-i n \varphi\right]
$$

where $\varphi$ is the toroidal angle, $\hat{E}(r, \theta)$ is the eigenmode envelope, and $\epsilon_{0}=r_{0} / R_{0}$ is determined by the local minimum of the potential

$$
V(r, \theta)=\frac{m^{2}}{r^{2}}\left(1+\epsilon_{0} \cos \theta\right)^{2}-\frac{\omega^{2}}{v_{A}^{2}(0)} \frac{n(r)}{n_{0}}(1+\epsilon \cos \theta)^{2}
$$

Substituting Eq. (14) into Eq. (6) we obtain the next order equation

$$
\left(\frac{1}{r} \frac{\partial}{\partial r} r \frac{\partial}{\partial r}-V(r, \theta)+2 \frac{i m}{r^{2}}\left(1+\epsilon_{0} \cos \theta\right) \frac{\partial}{\partial \theta}\right) \hat{E}(r, \theta)=0
$$

The potential well has a minimum at $\theta=0$ and $r=r_{0}$, which allows a radially and poloidally localized eigenmode if $\epsilon$ is not too small. As we will see later, $\epsilon=1 / 3$ is enough for CAE to be localized at the low field side in a region with $\theta<1$. Assuming that $\hat{E}(r, \theta)=\hat{E}_{0} X(r) Y(\theta)$, averaging Eq. (16) over the poloidal angle, and taking the limit $\hat{E}(r, \theta) \rightarrow 0$ as $\theta \rightarrow \pm \pi / 2$, weoobtain a radial envelope equation for $X(r)$, similar to Eq. (8). The solution is given by $X(r)=\phi_{s}\left(\sqrt{2}\left(r-r_{0}\right) / \Delta\right)$ with the eigenfrequency given by Eq. (10), where Alfvén velocity is evaluated at $r=r_{0}, \theta=0$ and

$$
\Delta^{2} / a^{2}=\sqrt{2 \sigma_{i} /\left(1+\sigma_{i}\right)} /\left[m\left(1+\sigma_{i}\right)\left(1+\epsilon_{0}\right)\right]
$$

The last term in the round brackets in Eq. (16) vanishes in the poloidal integration. It means that this term corresponds to a small contribution on the order of $1 / \sqrt{m}$ to the eikonal, Eq. (14), and a small frequency contribution on the order of $v_{A} / r \ll \omega$. To simplify further in the derivation we will neglect this term. Later we will show that our analytical solution agrees with the numerical solution of Eq. (16). After multiplying Eq. (6) by $X(r)$ and integrating over the minor radius, the poloidal envelope $Y(\theta)$ is determined by the following higher order equation in $1 / \mathrm{m}$

$$
\left(\frac{\partial^{2}}{\partial \theta^{2}}+\lambda+\frac{V\left(r_{0}, 0\right) r_{0}^{2}}{2} \frac{\epsilon_{0} \theta^{2}}{1+\epsilon_{0}}\right) Y(\theta)=0 .
$$

Note that the constant $\lambda$ is on the order of $m \theta^{2}$ and is introduced to provide a localized poloidal envelope solution. The contribution of $\lambda$ in the equation for $X(r)$ leads to a negligible term to 
the eigenfrequency on the order of $\theta^{2} v_{A} / r$. The localized solution at small poloidal eigenvalue $k$ is given by $Y(\theta)=\phi_{k}\left(\frac{\sqrt{2} \theta}{\Theta}\right)$, where $\Theta^{2}=\sqrt{4\left(1+\epsilon_{0}\right) /(2 s+1) \epsilon_{0}} \Delta / r_{0}$. For a localized eigenmode with $\Theta<1$, we have $\epsilon_{0}>4 / m$ for $\sigma_{i} \sim 1, s=0$. For typical poloidal mode numbers, $m>15$, it requires $\epsilon_{0}>1 / 4$ to have a localized poloidal envelope.

In summary, the localized CAE solution is given by

$$
E_{\theta}=\hat{E}_{0} \phi_{k}\left(\frac{\sqrt{2} \theta}{\Theta}\right) \phi_{s}\left(\frac{\sqrt{2}\left(r-r_{0}\right)}{\Delta}\right) \exp \left[-i \omega t+i m\left(\theta+\epsilon_{0} \sin \theta\right)-i n \varphi\right] .
$$

This analytical solution agrees well with the numerical solution of Eq. 16, which was obtained by using a shooting method with the boundary conditions: $\hat{E}(r, \theta)=0$ and $\partial \hat{E}(r, \theta) / \partial r=0$ at $r=1.3 a$. The results are presented in Fig.1, which shows a good agreement on the poloidal envelope solution for $\omega=3 \omega_{c D 0}, s=0, k=0, \sigma_{i}=0.2$ and $m=36$.

\section{CAE DAMPING}

\subsection{Perturbative Analysis}

Kinetic effects due to resonance interaction of thermal ions and electrons with CAE can be included perturbatively. These effects are described by the antihermitian part of the permeability tensor for each species $[9,12]$. We expand the perturbed electric field and the eigenfrequency as $\mathbf{E}=\mathbf{E}^{0}+\mathbf{E}^{1}$ and $\omega=\omega^{0}+\omega^{1}$, where $\mathbf{E}^{1}$ and $\omega^{1}$ are due to small kinetic corrections to the magnetohydrodynamic eigenmode and eigenfrequency. Then, from Eq. (1) the expression for the growth rate is given by

$$
\gamma=-\sum_{j} \operatorname{Im} \int \mathbf{E}^{*} \cdot \hat{\boldsymbol{\epsilon}}_{j}^{A} \cdot \mathbf{E} d^{3} r / \int \mathbf{E}^{*} \cdot \frac{1}{\omega^{2}} \frac{\partial \omega^{2} \hat{\boldsymbol{\epsilon}}}{\partial \omega} \cdot \mathbf{E} d^{3} r
$$

Further we will neglect the small correction to the perturbed electric field $\mathbf{E} \simeq \mathbf{E}^{0}$. The radial component of the perturbed electric field is related to the poloidal component by

$$
E_{r}=-\frac{i}{\lambda} E_{\theta} \equiv \frac{F}{H} E_{\theta}
$$

which results from Eqs. (4) in the leading order of $1 / \mathrm{m}$.

\subsection{CAE-particle Resonance}

The electron transit time damping of CAE is not important when the number of resonant electrons is small, i.e., when the parallel phase velocity exceeds the electron thermal velocity $v_{T e}$. The main resonance contribution to the electron permeability tensor is given by [12]

$$
\hat{\epsilon}_{e r r}^{A}=i \sqrt{\pi} \beta_{e} \frac{c^{2} k_{\perp}^{2}}{\omega^{2}} \zeta_{e} e^{-\zeta_{e}^{2}}
$$


where $\zeta_{e}=\omega /\left|k_{\|}\right| v_{T e}, \beta_{e}=8 \pi n_{e} T_{e} / B^{2}, k_{\|} \simeq m\left(1+\epsilon_{0}\right) / q R_{0}-n / R_{0}$, and $q$ is the safety factor. Then, in the limit $\omega^{2} \gg \omega_{c i}^{2}$ the electron Landau damping rate is given by

$$
\gamma_{e} \simeq-\omega \beta_{e} \zeta_{e} e^{-\zeta_{e}^{2}}
$$

The thermal ions contribute to the damping through the cyclotron resonances. Considering the limits of $b_{i}=k_{\perp}^{2} v_{T i}^{2} / 2 \omega_{c i}^{2} \sim \omega^{2} v_{T i}^{2} / \omega_{c i}^{2} v_{A}^{2} \ll 1, \omega_{c i} /\left|k_{\|}\right| v_{T i} \gg 1$, where $v_{T i}=\sqrt{2 T_{i} / m_{i}}$ is the ion thermal velocity, the antihermitian part of the ion permeability tensor has the form [12]

$$
\hat{\epsilon}_{i r r}^{A}=i \hat{\epsilon}_{i \theta r}^{A}=-i \hat{\epsilon}_{i r \theta}^{A}=\hat{\epsilon}_{i \theta \theta}^{A}=\sum_{l} g_{i l} \frac{l \omega_{c i} \sqrt{2}}{\left|k_{\|}\right| v_{T i}} \frac{Z_{p}\left(\zeta_{i l}\right)}{\zeta_{i l}}
$$

where $\zeta_{i l}=\sqrt{2}\left(\omega-l \omega_{c i}\right) /\left|k_{\|}\right| v_{T i}, Z_{p}$ is the plasma dispersion function, and

$$
g_{i l}=\frac{\omega_{p i}^{2}}{\omega \omega_{c i}}\left(\frac{b_{i}}{2}\right)^{l-1} \frac{e^{-b_{i}}}{2(l-1) !} .
$$

The thermal ion damping rate can be obtained from the energy transfer rate from the mode to the thermal ions. Integrating over the the mode localization region, we have

$$
\operatorname{Im} \sum_{i}<\mathbf{E}\left|\hat{\boldsymbol{\epsilon}}_{i}^{A}\right| \mathbf{E}>\equiv \operatorname{Im} \sum_{i} \int \mathbf{E}^{*} \cdot \hat{\boldsymbol{\epsilon}}_{i}^{A} \cdot \mathbf{E} d^{3} r=2 \pi \int E_{\theta}^{2}(1-1 / \lambda)^{2} \operatorname{Im} \sum_{i} \hat{\epsilon}_{i \theta \theta}^{A} R d R d Z,
$$

where $Z$ is the coordinate along the major axis of the torus, $\lambda$ is the electric field polarization given by Eq. (21). Note from the form of the variable $\zeta_{i l}$, the tensor $\hat{\epsilon}_{i}^{A}$ is nonvanishing in the vicinity of the resonance surface $R=R_{c}$ determined by the resonance condition $\omega=l \omega_{c i}\left(R_{c}\right)$. The width of the resonance layer is on the order of $R\left|k_{\|}\right| v_{T i} / l \omega_{c i} \ll a$. Assuming that the plasma parameters and the perturbed electric field envelope in the resonance layer change slowly we can perform the integration over $R$ in Eq. (25) and we have

$$
\operatorname{Im} \sum_{i}<\mathbf{E}\left|\hat{\epsilon}_{i}^{A}\right| \mathbf{E}>=-\left.2 \pi^{2} R_{c}^{2} \int d Z E_{\theta}^{2}(1-1 / \lambda)^{2} \sum_{i l} g_{i l \mid}\right|_{R=R_{c}} .
$$

When the cyclotron resonance layer intersects the eigenmode location, the thermal ion cyclotron damping rate is maximum and is given by

$$
\gamma_{i} \simeq-\frac{a}{\epsilon \Delta} \frac{\omega^{3} v_{A}^{2}}{\bar{\omega}_{c}^{2} c^{2}} \sum_{i l} g_{i l}
$$

where $\Delta$ is given by Eq. (17), and $d Z=r d \theta$ is assumed. 


\subsection{CAE Damping Rates}

To drive the CAE unstable the fast ion growth rate must be higher than the total damping rate. The CAE can be most unstable if the damping rate is smallest. The electron Landau damping can be minimized by choosing modes with phase velocity higher than the thermal electron velocity, i.e., $k_{\|} \ll \omega / v_{T e}[11,12]$. The ion cyclotron damping is significant if ions are in cyclotron resonance with the mode and if the cyclotron harmonic number is not high. Therefore, eigenmodes localized near the plasma edge and with eigenfrequencies corresponding to low multiples of the edge thermal ion cyclotron frequency can be weakly damped due to low edge plasma ion density and temperature. For higher cyclotron harmonics with $l=\omega / \omega_{c i}>5$ the thermal ion damping is exponentially small.

The damping rate are computed for typical TFTR supershot plasma parameters [14]: $R_{0}=$ $2.52 m, a=0.9 m$, the safety factor profile $q(r)=q_{0} /\left[1-1.32 r^{2} / a^{2}+0.54 r^{4} / a^{4}\right]$ for $r \leq a$, $q_{0}=0.85$, the toroidal magnetic field at magnetic axis $B_{0}=4.75 T$, the bulk plasma density profile is given by Eq. (7) with $\sigma_{i}=0.2$, the temperature profiles $T_{i}(r)=2 T_{e}(r)=10 \times(1-$ $\left.r^{2} / a^{2}\right)^{2} \mathrm{keV}$. We consider plasmas with deuterium and tritium ions. For neutral beam ions we assume a Maxwellian velocity distribution function with the temperature profile $T_{b i}(r)=50 \times$ $\left(1-r^{2} / a^{2}\right)^{1.5} \mathrm{keV}$ and the density profile $n_{b i}(r)=n_{b i 0}\left(1-r^{2} / a^{2}\right)^{\sigma_{b}}$, where $\sigma_{b}=1$. For DT plasmas with deuterium and tritium beams the damping rates are given in Fig. 2 for $n_{D 0}=n_{T 0}=10 \times$ $n_{b D 0}=10 \times n_{b T 0}=0.15 \times 10^{14} \mathrm{~cm}^{-3}$. Here we choose $m \sim n$. As expected, CAE with frequencies equal to harmonics of the edge deuterium cyclotron frequency have negligible damping rate and can be most easily excited by fast particles. For eigenmodes with $\omega<2 \omega_{\text {cTedge }}$ there is no thermal ion cyclotron damping in our model because the perturbed electric field rotates in the electron cyclotron gyration direction. The cyclotron damping in this range of frequencies is mainly due to the NBI ions which have a slowing down anisotropic velocity distribution function [14]. The resonance layer for beam ion - CAE cyclotron resonance can have a finite width due to the Doppler frequency shift, which produces a perturbed electric field component that rotates in the ion cyclotron gyration direction and will lead to a finite cyclotron damping.

In Fig. 3 we present the damping rates for a pure tritium thermal plasma and tritium beams with $n_{T 0}=10 \times n_{b T 0}=0.3 \times 10^{14} \mathrm{~cm}^{-3}$. As expected from Eq. (24), at fixed eigenfrequency the tritium cyclotron damping rate is less than the deuteron cyclotron damping rate because of lower tritium cyclotron frequency (or higher tritium cyclotron harmonic number). From Fig.3 one can see that in tritium plasmas, CAE eigenmodes with frequency at edge tritium cyclotron harmonics can be more easily excited.

Note that by assuming the ACI growth rate to be a slowly varying function of frequency (see [8]) the emission spectra due to the ACI should consist of two distinct parts. The low frequency part contains sharp peaks with widths proportional to the growth rates or to the density of fast particles. The high frequency broadband part with $\omega>5 \omega_{c \text { Dedge }}$ is determined mainly by the fast ion driving mechanism because of exponentially small thermal plasma damping. Due to high velocity of fast particles and finite $k_{\|}$, and thus high Doppler frequency shift to the cyclotron 
resonance, the neighboring fast particle-mode cyclotron resonances will be easily overlapped. The thermal ion damping will contribute to a slight modulation in the broadband spectrum.

\section{ICE SPECTRUM}

\subsection{Basic Equations}

To understand the ICE spectra we consider the ACI driven by charged fusion products based on the formalism developed in Ref.[8]. Then, the fast particle resonance contribution to the anti-hermitian part of the dielectric permiability tensor is given by

$$
\begin{aligned}
<\mathbf{E}\left|\hat{\boldsymbol{\epsilon}}_{f}^{A}\right| \mathbf{E}> & =-i \sum_{l, \sigma} \frac{4 \pi^{2} e_{f} c}{\omega} \times \\
& \times\left.\int d P_{\varphi} d \mathcal{E} d \mu I^{2} \mathbf{G}_{l}^{\prime *} \cdot \mathbf{E}^{*} \mathbf{G}_{l} \cdot \mathbf{E}\left[\frac{\partial}{\partial \mathcal{E}}+\frac{l \omega_{c f}}{\omega B} \frac{\partial}{\partial \mu}\right] f_{f}\right|_{\theta_{r \in s}\left(P_{\varphi}, \mathcal{E}, \mu, \sigma\right)},
\end{aligned}
$$

where the subscript $f$ denotes the fast particles, $P_{\varphi}, \mathcal{E}, \mu$ are adiabatic invariants, $P_{\varphi}=e_{f} \psi /$ $2 \pi m_{f} c-v_{\|} R, \mathcal{E}=v^{2} / 2, \mu=v_{\perp}^{2} / 2 B, \psi$ is the poloidal magnetic flux, $\sigma$ is the sign of the parallel velocity, vectors $\mathbf{G}_{l}$ and $\mathbf{G}_{l}^{\prime}$ have components in $r, \theta, \varphi$ coordinate system with $\mathbf{G}_{l}=$ $\left\{-i v_{\perp} \partial J_{l} / \partial z ; v_{\perp} \omega J_{l} /\left(\omega_{c} z\right) ; v_{\|} J_{l}\right\}$ and $\mathbf{G}_{l}^{\prime}=\left\{-i v_{\perp} \partial J_{l} / \partial z ; v_{\perp} l J_{l} / z ; v_{\|} J_{l}\right\}$, respectively, $J_{l}=J_{l}(z)$ is the $l-t h$ order Bessel function of the first kind, $z=k_{\perp} v_{\perp} / \omega_{c f}, f_{f}=f_{f}\left(P_{\varphi}, \mathcal{E}, \mu, \sigma\right)$ is the fast particle equilibrium distribution function. All quantities in Eq. (28) are taken at the resonance point on the drift trajectory of fast particles with $\theta=\theta_{\text {res }}\left(P_{\varphi}, \mathcal{E}, \mu, \sigma\right)$ determined by the resonance condition

$$
\omega-l \omega_{c f}(r(\theta), \theta)-\omega_{D f}(r(\theta), \theta)=0
$$

where $r(\theta)$ describes the fast particle drift orbit. Also we let

$$
\omega_{D f}=\mathbf{k} \cdot\left(\mathbf{v}_{\|}+\mathbf{v}_{d f}\right) \simeq k_{\|} \sigma \sqrt{2 \mathcal{E}} \sqrt{1-\mu B / \mathcal{E}}-\frac{2 m}{r^{2}} \frac{\mathcal{E}}{\omega_{c 0}}\left(\frac{R}{R_{0}}-1\right)\left(1-\frac{\mu B}{2 \mathcal{E}}\right)
$$

where $\mathbf{v}_{d f}$ is the magnetic drift velocity. Also, $I^{2}$ in Eq. (28) is given by

$$
I^{2}=8 \pi\left\{\left|d\left(l \omega_{c f}+\omega_{D f}\right) / d t\right|^{3}+C\left[d^{2}\left(l \omega_{c f}+\omega_{D f}\right) / d t^{2}\right]^{2}\right\}^{-1 / 3}
$$

where $C=2.6943$, the time derivatives are taken along the fast particle drift trajectory.

Eq. (28) includes fast particle FLR effects and is obtained by integrating the high frequency gyrokinetic equation along the particle orbit. $\omega_{D f}$ presents the Doppler shift due to the parallel and drift motion. The term $I$ has the time dimension and gives the time of fast particle eigenmode resonance interaction during one pass through the resonance layer. The sign of the growth rate (Eq. (20) and Eq. (28)) depends on the signs of $\mathcal{E}$ and $\mu$ derivatives of the distribution 
function and the weights with which these derivatives are integrated over the phase space. These weights are given by three terms. The first is driven by FLR effects, i.e., $\mathbf{G}_{l}$ and $\mathbf{G}_{l}^{\prime}$ vectors which are the same as in the local theory. The second term $I$ is pure nonlocal. And the third term is related to the two- dimensional structure of the perturbed electric field E. Typically this term gives a comparable contribution to the instability in comparison with the FLR driven term. It allows an instability even for $v_{f 0} \leq v_{A}$, where $v_{f 0}$ is the fast particle birth velocity.

The plasma inhomogeneity and finite banana width can lead to a large velocity space anisotropy of fast particle distribution function. The anisotropy is higher near the plasma periphery on the low field side, where the spatial gradient of fast fusion products is higher [15]. The edge fast particles are mainly barely trapped particles which come from the plasma center and have the highest radial excursion from the magnetic surface. The fast charged fusion product distribution can be expressed as

$$
f_{f}=n_{f}\left(P_{\varphi}\right) f_{v}(v) \delta\left(\frac{\mu B_{0}}{\mathcal{E}}-\Lambda_{0}\right)
$$

where $f_{v}(v)$ represents the velocity distribution, and $\Lambda_{0}$ is the pitch angle of barely trapped particles. $\Lambda_{0}$ can be obtained from the equation $\left(e_{f} / 2 \pi m_{f} c\right) \psi(R, Z)_{\mid R=\Lambda_{0} R_{0}, Z=0}=P_{\varphi}$. Integrating Eq. (28) by parts and using Eq. (32) we obtain

$$
\begin{aligned}
& <\mathbf{E}\left|\hat{\boldsymbol{\epsilon}}_{f}^{A}\right| \mathbf{E}>=-i \sum_{l, \sigma} \frac{4 \pi^{2} e_{f}^{2} B}{m_{f} \omega_{c f} \omega} \int d P_{\varphi} \times \\
& \times\left.\left\{F\left(\mathcal{E}_{2}\right)-F\left(\mathcal{E}_{1}\right)-\int d \mathcal{E} n_{f} f_{v} \frac{\mathcal{E}}{B_{0}}\left[\frac{\partial}{\partial \mathcal{E}}+\frac{l \omega_{c \alpha}}{\omega B} \frac{\partial}{\partial \mu}\right] I^{2} \mathbf{G}_{l}^{\prime *} \cdot \mathbf{E}^{*} \mathbf{G}_{l} \cdot \mathbf{E}\right\}\right|_{\mu=\Lambda_{0} \mathcal{E} / B_{0}},
\end{aligned}
$$

where $F=\left(1+l \omega_{c \alpha} \mathcal{E} /(\omega B \mu)\right) n_{f} f_{v} I^{2} \mathbf{G}_{l}^{\prime *} \cdot \mathbf{E}^{*} \mathbf{G}_{l} \cdot \mathbf{E}\left(\mathcal{E} / B_{0}\right), \mathcal{E}_{1,2}=\mathcal{E}_{1,2}\left(P_{\varphi}\right)\left(\mathcal{E}_{1}<\mathcal{E}_{2}\right)$ are the energy limits determined from the resonance condition Eq. (29) at fixed $P_{\varphi}$. The differential operators in Eq. (33) operate on all quantities to their right hand side. These quantities are taken at the resonance point $\theta=\theta_{\text {res }}\left(P_{\varphi}, \mathcal{E}, \mu, \sigma\right)$.

\subsection{ACI Growth Rate}

The numerical results of ACI growth rates are presented for JET[1] and TFTR[14] DT plasmas. The fast particle density profile is chosen as $n_{f}=n_{f 0}\left(1-P_{\varphi} / \bar{\psi}\right)^{3.75}[14]$, where $n_{f 0}$ is the central fast particle density, $\bar{\psi}=e_{f} \psi_{l} / 2 \pi m_{f} c$, and $\psi_{l}$ is the poloidal magnetic flux at the plasma surface. Because the ACI growth rate depends linearly on the fast particle density at the CAE localization region, the effect of fast particle profiles will only modify the growth rate slightly. The velocity distribution is chosen as in Ref.[8]

$$
f_{v}=\frac{4 B_{0}}{B v_{0}^{2}\left(v_{T-}+v_{T+}\right) \sqrt{\pi}} \sqrt{1-\Lambda_{0} \frac{B}{B_{0}}} \sum_{+,-} \exp \left(-\frac{\left(v-v_{0}\right)^{2}}{v_{T \pm}^{2}}\right) \eta\left( \pm\left(v-v_{0}\right)\right)
$$


where $v_{T-}$ is an adjustable parameter, $v_{T+}=\sqrt{2 T_{i} /\left(m_{1}+m_{2}\right)}, m_{1}$ and $m_{2}$ are the mass of reacting nuclei, $T_{i}$ is the temperature taken to be $50 \mathrm{keV}$ for beam heated plasmas, and $\eta$ is the Heaviside step function. This distribution function is typical during the initial stage of NBI heated TFTR discharges. The velocity spread of the fast particle distribution function is due to the thermal velocity spread of the reacting nuclei. The positive velocity gradient of the fast particle distribution may also result from anomalous fast particle losses caused by MHD magnetic perturbations or toroidal ripples. As noted earlier [8, 9], the thermal spread is a stabilizing factor for the ACI. Therefore, ICE observed in the steady state phase of tokamak discharges may indicate an anomalous fast particle loss.

Consider the JET DT discharge \#26148 at $13.2 s$ with edge plasma profiles given by TRANSP simulations[1], $R_{0}=2.96 \mathrm{~m}, a=1.2 \mathrm{~m}, B_{0}=2.8 T$, and the safety factor profile $q(r)=q_{0} /[1-$ $\left.1.21 r^{2} / a^{2}+0.38 r^{4} / a^{4}\right]$ for $r \leq a, q_{0}=0.9$. For the bulk deuterium and tritium plasmas their densities are $n_{D 0}=10 \times n_{T 0}=0.3 \times 10^{14} \mathrm{~cm}^{-3}$ with density profiles given by Eq. (7) with $\sigma_{i}=0.3$, and their temperature profiles are given in section 3.3 with $T_{i}(0)=10 \mathrm{keV}$; andT $(0)=7 \mathrm{keV}$. For the beam components, $n_{b D 0}=10 \times n_{b T 0}=0.1 \times n_{D 0}$, and for fusion protons and $\alpha$-particles $n_{\alpha 0}=30 \times n_{p 0}=2 . \times 10^{10} \mathrm{~cm}^{-3}$. Other parameters are taken to be similar to those in section 3.3.

Presented in Fig. 4 are the ACI growth rates driven by $\alpha$-particles (solid curve) with $v_{T-}=$ $v_{T+}=0.107 v_{\alpha 0}$ and by fusion protons (dashed curve) with $v_{T-}=v_{T+}=0.058 v_{p 0}$. For each frequency the two-dimensional eigenmode structure is obtained with the analysis of Section 2 . The toroidal mode number $n$ is varied to find the ACI with the maximum growth rate. The upper $n$ limit is determined by the electron damping. Typically $n \sim m$, which provides enough Doppler shift for the protons with birth velocity $v_{f 0}=2.4 \times 10^{9} \mathrm{~cm} / \mathrm{sec}$ to excite the instability at half edge proton cyclotron harmonics. It require thats $k_{\|} \geq \omega_{c D e d g e} / v_{p 0} \simeq 3.5 m^{-1}$ for JET and $k_{\|} \geq 7 m^{-1}$ for TFTR. Note that at the edge $q \geq 4$ and $k_{\|} \simeq-n / R$. In JET DT plasmas the tritium density is small, and the ion cyclotron damping is mainly due to deuterium, and therefore ICE has peaks at the harmonics of deuterium edge cyclotron frequency. The ACI growth rates presented in Fig. 4 are consistent with the JET ICE spectrum. The proton driven ACI has lower growth rates than the $\alpha$ driven ACI by the density ratio of the $\alpha$-particle to the proton, and thus the neutron emission rate ratio of DD to DT plasmas.

Fig. 5 presents growth rates of the ACI driven by $\alpha$-particles and protons for the TFTR DT plasma (shot \#73255) at the time $250 \mathrm{~ms}$ after the start of NBI[4]. The central ion densities are $n_{D 0}=n_{T 0}=0.15 \times 10^{14} \mathrm{~cm}^{-3}$ for thermal ions, $n_{b D 0}=n_{b T 0}=0.1 \times n_{D 0}$ for deuterium and tritium beam ions, and $n_{\alpha 0}=50 \times n_{p 0}=2 \times 10^{10} \mathrm{~cm}^{-3}$ for fusion protons and $\alpha$-particles with $v_{T-}=v_{T+}$ for both groups of particles. The rest of plasma parameters are listed in Section 3.3. Fig. 6 presents the ACI growth rates for DT discharge with the same plasma parameters as in Fig.5, but with a broader fast particle velocity distribution $v_{T-}=2 v_{T+}$. Comparison of Figs. 5 and 6 shows that in TFTR the ACI growth rates are more sensitive to the velocity spread of the distribution function of $\alpha$-particles than protons, where $v_{\alpha 0}<v_{A}$ and $v_{p 0}>v_{A}$. In steady state the fusion reaction saturates, and the fast products has a classical slowing-down distribution in energy. However, the anisotropy of the fusion product distribution can still lead to ACI, but 
with lower growth rates. Therefore, in TFTR DD and DT steady state plasmas the ICE signal will be proportional to the neutron emission rate.

In contrast with Ref.[8] we have obtained the ACI driven by $\alpha$-particles at high cyclotron harmonics. The poloidally localized CAE provides conditons for the ACI to be excited by a single cyclotron resonance. Without the poloidal localization, neighboring cyclotron resonances have a stabilizing effect on ACI. Finally, in Fig.7 we present the ACI growth rates driven by $\alpha$-particles in TFTR DT shot \#73273 at the time $250 \mathrm{~ms}$ after the start of tritium NBI[4]. The plasma is characterized by a large tritium population with the central thermal tritium density $n_{T 0}=0.3 \times 10^{14} \mathrm{~cm}^{-3}$, the central tritium beam density $n_{b T 0}=0.1 \times n_{T 0}$, the central $\alpha$-particle density $n_{\alpha 0}=2 \times 10^{10} \mathrm{~cm}^{-3}$, and $v_{T-}=v_{T+}$. The contribution from the thermal deuterium is small and will be neglected. Modes with frequency at low number harmonics of edge tritium cyclotron frequency are least damped by the thermal tritium ions and will be unstable.

\section{CONCLUSIONS}

A two-dimensional analysis of compressional Alfvén eigenmode has been performed. The analytical results are in good agreement with the numerical solutions of the two-dimensional eigenmode equation. The eigenmode solution is characterized by an envelope localized in both the poloidal and radial directions at the low magnetic field side of the plasma edge. Twodimensional CAE solutions have been employed to calculate the ACI growth rates driven by fusion products to explain the experimentally ICE spectra.

We have shown that the thermal ion cyclotron damping determines the form of ICE spectra. The ACI growth rates for deuterium and tritium plasmas are consistent with the experimentally observed ICE spectra. In DD plasmas odd deuterium harmonics are excited by protons due to the Doppler shifted cyclotron resonance. The growth rates are proportional to the density of fast particles, which is consistent with ICE peak signal amplitudes and the neutron emission rate.

The low frequency part of ACI (and thus ICE) spectrum contains sharp peaks with widths proportional to the ACI growth rates or the density of fast particles. The high frequency broadband part with $\omega>5 \omega_{c D e d g e}$ is determined mainly by the Doppler shifted fast particle - CAE resonance because of exponentially small thermal plasma dampings. Due to high velocity of fast particles and finite $k_{\|}$(i.e., high Doppler frequency shift to the cyclotron resonance) the fast particle cyclotron resonances with the neighboring modes will overlap. The theoretical results of ACI spectra agree with ICE observations.

Based on the studies, several issues can raised for experimental checks. First, the ICE amplitude can be increased by increasing the population of barely trapped fast particles by the adiabatic compression in major radius. The adiabatic compression provides the transition from confined counter-passing particles to barely trapped particles[16]. The second is the excitation of the loss-cone instability for the anomalous scattering of confined counter-passing particles. The destabilizing factor is due to the counter-injection of tritium beams which allow a positive shift of $\alpha$-particle parallel velocity at the plasma edge. 
Finally we emphasize the importance of ICE phenomena in the charged fusion product particle physics for the purpose of the diagnostic of edge plasma parameters and for studying the channeling of $\alpha$-particle energy to thermal ions through ACI.

\section{ACKNOWLEDGMENTS}

This work was supported by the United States Department of Energy under Contract No. DE-AC02-CHO-3073. 


\section{References}

[1] Cottrell, G. A., et.al., Nucl. Fusion 33 (1993) 1365.

[2] Cottrell, G. A., Dendy, R. O., Phys. Rev. Lett. 60 (1988) 33 .

[3] Greene, G. J., et.al., in Proceedings of 17th European Conference on Controlled Fusion and Plasma Heating, Amsterdam, Netherlands, 1990, edited by G. Briffod, Adri Nijsen-Vis, and F. C. Shüller (European Physical Society, Petit-Lancy, Switzerland, 1990), Part IV, Vol. 14B, p.1540.

[4] Cauffman, S., Majeski, R., Rev. Sci. Instrum 66 (1995) 817.

[5] Dendy, R. O., et.al., Phys. Plasmas 1 (1994) 1918.

[6] Belikov, V. S., Kolesnichenko, Ya. I., Fusion Technol. 25 (1994) 258.

[7] Coppi, B., Phys. Lett. A 172 (1993) 439.

[8] Gorelenkov, N. N., Cheng, C. Z., to appear in Phys. Plasmas 1 (1995).

[9] Mikhailovskii, A. B., in Reviews of Plasma Physics, edited by M. A. Leontovich (Consultants Bureau, New York, 1986), Vol.9, p.103.

[10] Belikov, V.S., et.al., this proceedings.

[11] Coppi, B., et.al., Phys. Fluids 29 (1986) 4060.

[12] Akhiezer, A. I., et.al., Plasma Electrodynamics (Pergamon Press, Oxford, New York, 1975), Vol.1.

[13] Mahajan, S. M., Ross, D. W., Phys. Fluids 26 (1983) 2561.

[14] Budny, R. V., et.al., Nucl. Fusion 32 (1992) 429.

[15] Gorelenkov, N. N., Sov. J. Plasma Phys. 18 (1992) 289.

[16] Herrman, H. W., et.al. this proceedings. 
Figure 1: The CAE poloidal envelope at $r=r_{0}$, which corrsponds to the minimum of the potential given by Eq.15. The numerical eigenmode solution is presented by the real and imaginary parts of the eigenmode envelope.

Figure 2: Ion cyclotron (solid curve) and electron Landau (dashed curve) damping rates of CAE versus eigenfrequency in a deuterium-tritium plasma with the thermal ion density profile $n_{D}(r)=n_{T}(r)=0.15 \times 10^{14}\left(1-r^{2} / a^{2}\right)^{0.2} \mathrm{~cm}^{-3}$, and the neutral beam injected DT ion density $n_{b D}(r)=n_{b T}(r)=0.5 \times 10^{13}\left(1-r^{2} / a^{2}\right)^{0.2} \mathrm{~cm}^{-3}$. Other plasma parameters are: $B_{0}=4.75 T$, $R_{0}=2.52 m, a=0.9 m$, the safety factor profile $q(r)=q_{0} /\left[1-1.32 r^{2} / a^{2}+0.54 r^{4} / a^{4}\right]$, for $r \leq a$, $q_{0}=0.85$, the bulk plasma temperature profiles $T_{D}(r)=2 T_{e}(r)=10 \times\left(1-r^{2} / a^{2}\right)^{2} \mathrm{keV}$, and the beam ion temperature profile $T_{b D}(r)=50 \times\left(1-r^{2} / a^{2}\right)^{2} k e V$.

Figure 3: Ion cyclotron (solid curve) and electron Landau (dashed curve) damping rates versus eigenfrequency in a tritium plasma with the density profile $n_{T}(r)=0.3 \times 10^{14}\left(1-r^{2} / a^{2}\right)^{0.2} \mathrm{~cm}^{-3}$, and NBI tritium ion density profile $n_{b T}(r)=0.3 \times 10^{13}\left(1-r^{2} / a^{2}\right) \mathrm{cm}^{-3}$. Other plasma parameters are the same as in Fig.2.

Figure 4: The ACI growth rates driven by protons (dashed curve) and $\alpha$-particles (solid curve) for JET DT plasmas. The velocity distribution functions of fast particles are given by Eq. (34), where for protons $v_{T-}=v_{T+}=0.058 v_{p 0}$, and for $\alpha$-particles $v_{T-}=v_{T+}=0.107 v_{\alpha 0}$. Fast particle densities are $n_{\alpha 0}=30 \times n_{p 0}=2 . \times 10^{10} \mathrm{~cm}^{-3}$. The thermal ion density profiles are $n_{D}(r)=10 \times n_{T}(r)=0.3 \times 10^{14}\left(1-r^{2} / a^{2}\right)^{0.3} \mathrm{~cm}^{-3}$. Other plasma parameters are: $B_{0}=4.75 T$, $R_{0}=2.96 m, a=1.2 m$, the safety factor profile $q(r)=q_{0} /\left[1-1.21 r^{2} / a^{2}+0.38 r^{4} / a^{4}\right]$ for $r \leq a, q_{0}=0.9$, and the bulk plasma temperature profiles atdt2432926 $T_{i}(r)=1.43 \times T_{e}(r)=$ $10 \times\left(1-r^{2} / a^{2}\right)^{2} \mathrm{keV}$.

Figure 5: The ACI growth rates driven by protons (dashed curve) and $\alpha$-particles (solid curve) for TFTR DT plasmas. The velocity distribution functions of fast particles are given by Eq. (34), where for protons $v_{T-}=v_{T+}=0.058 v_{p 0}$, and for $\alpha$-particles $v_{T-}=v_{T+}=0.107 v_{\alpha 0}$. Fast particle densities are $n_{\alpha 0}=50 \times n_{p 0}=2 . \times 10^{10} \mathrm{~cm}^{-3}$. Other parameters are the same as in Fig.2

Figure 6: The ACI growth rates driven by protons (dashed curve) and $\alpha$-particles (solid curve) for TFTR DT plasmas, but with wider velocity spread in the fast particle distribution functions: for protons $v_{T-}=2 v_{T+}=0.116 v_{p 0}$, and for $\alpha$-particles $v_{T-}=2 v_{T+}=0.214 v_{\alpha 0}$.

Figure 7: The ACI growth rates driven by $\alpha$-particles for TFTR DT plasmas. The frequency spectra peaks are at harmonics of edge tritium cyclotron frequency due to negligible deuterium damping. The fast particle velocity distribution functions are given by Eq. (34) with $v_{T-}=$ $v_{T+}=0.107 v_{\alpha 0}$. Other parameters are the same as in Fig.5 


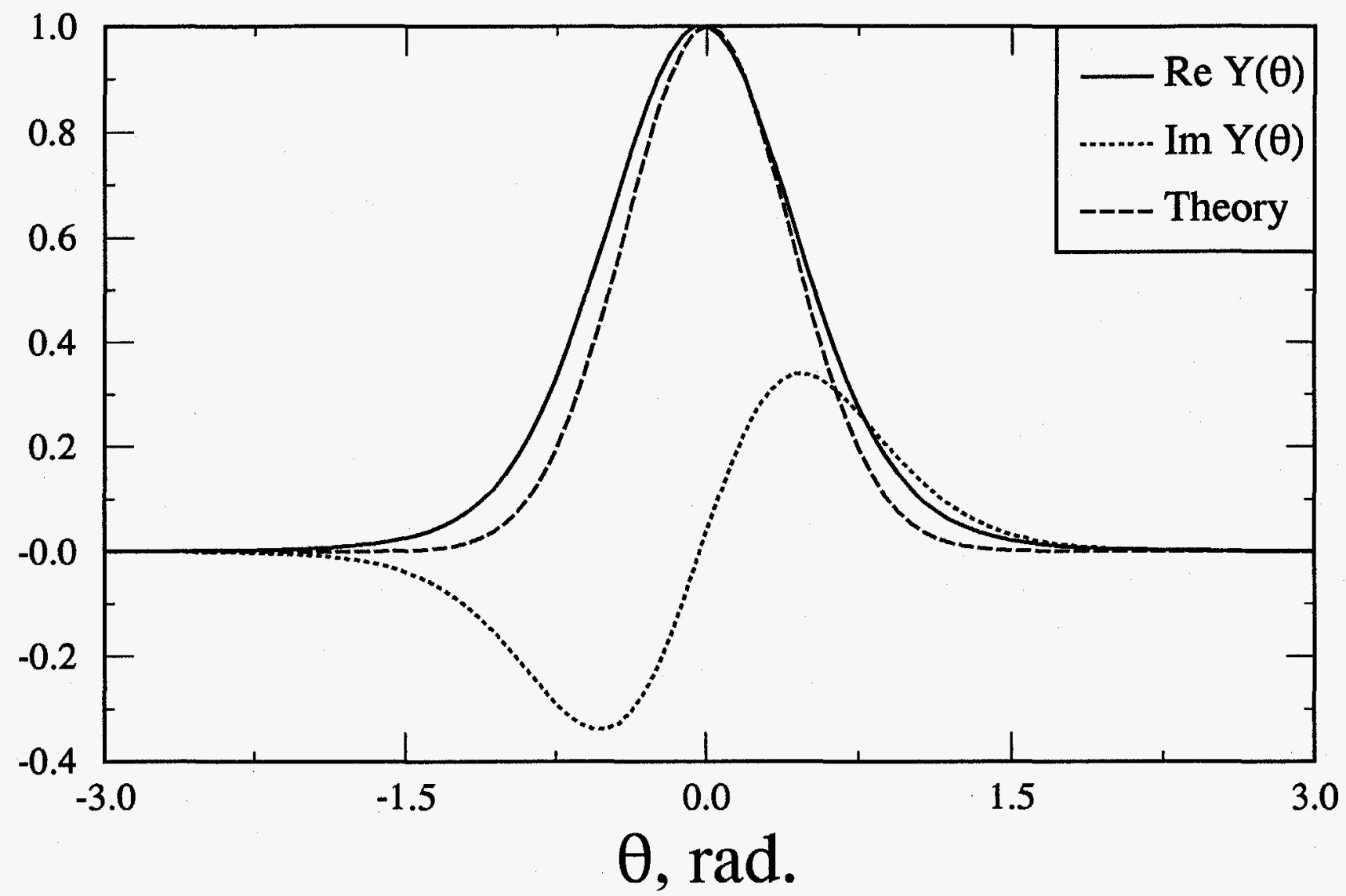

Fig. 1. 


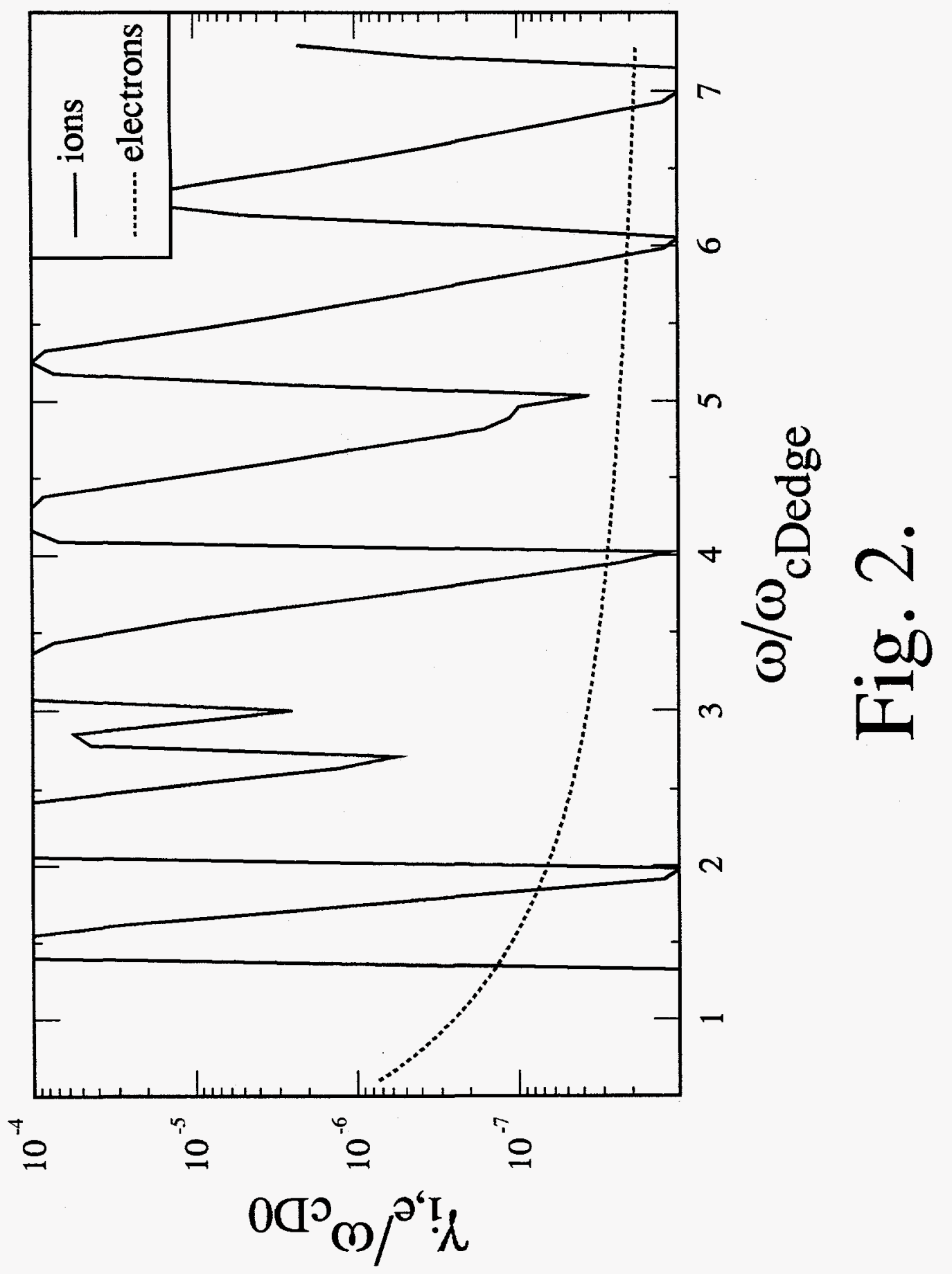




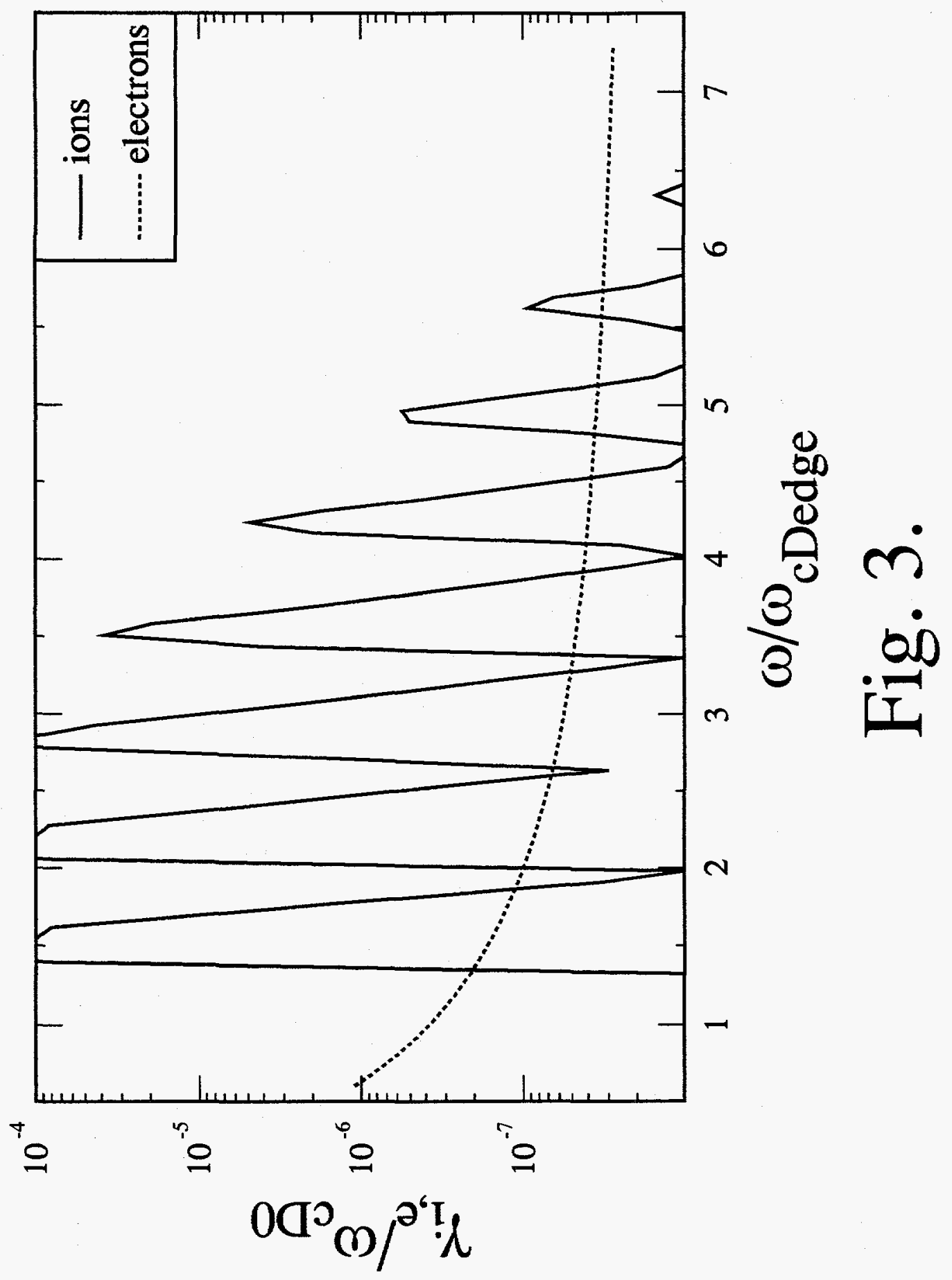




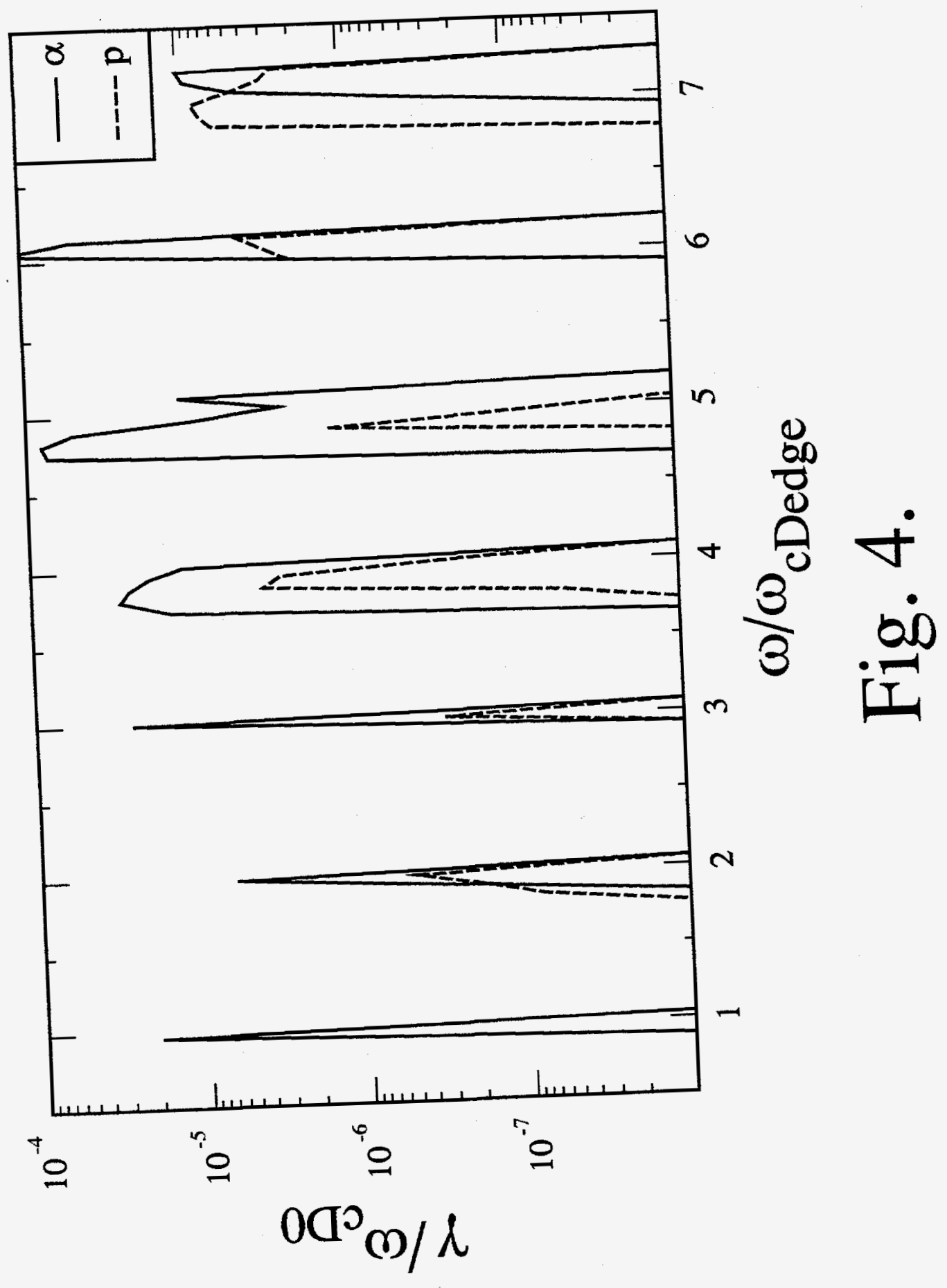




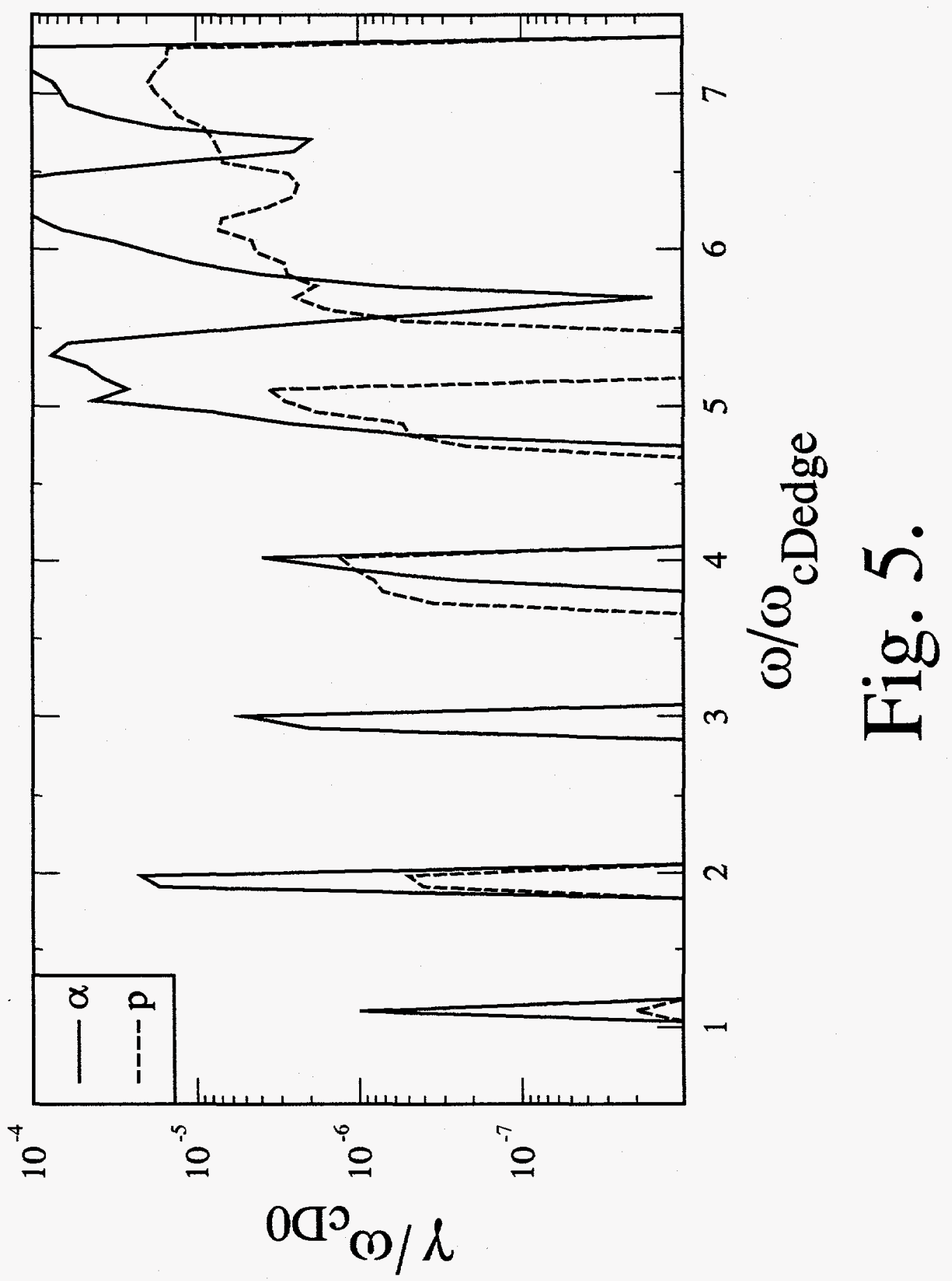




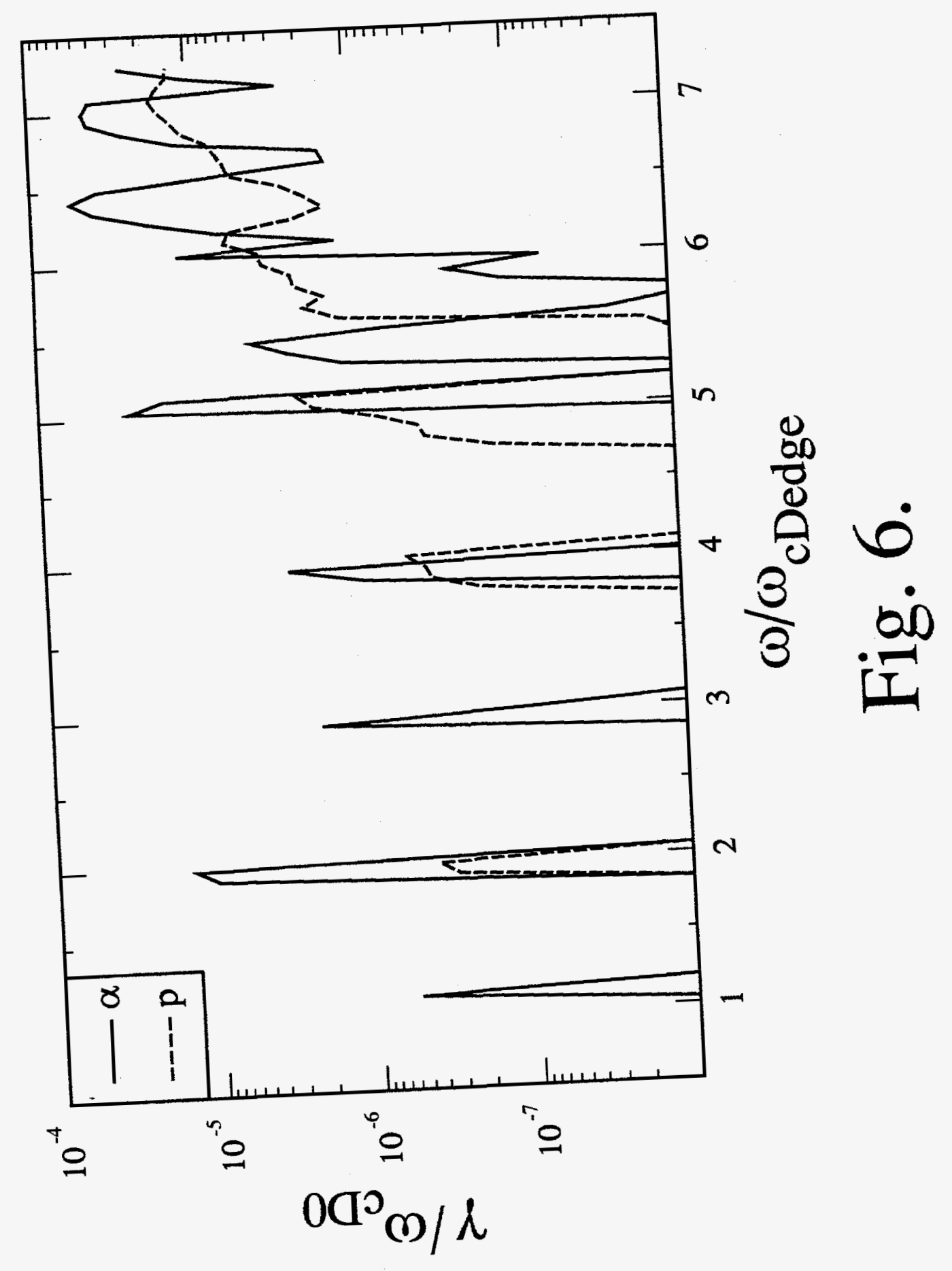




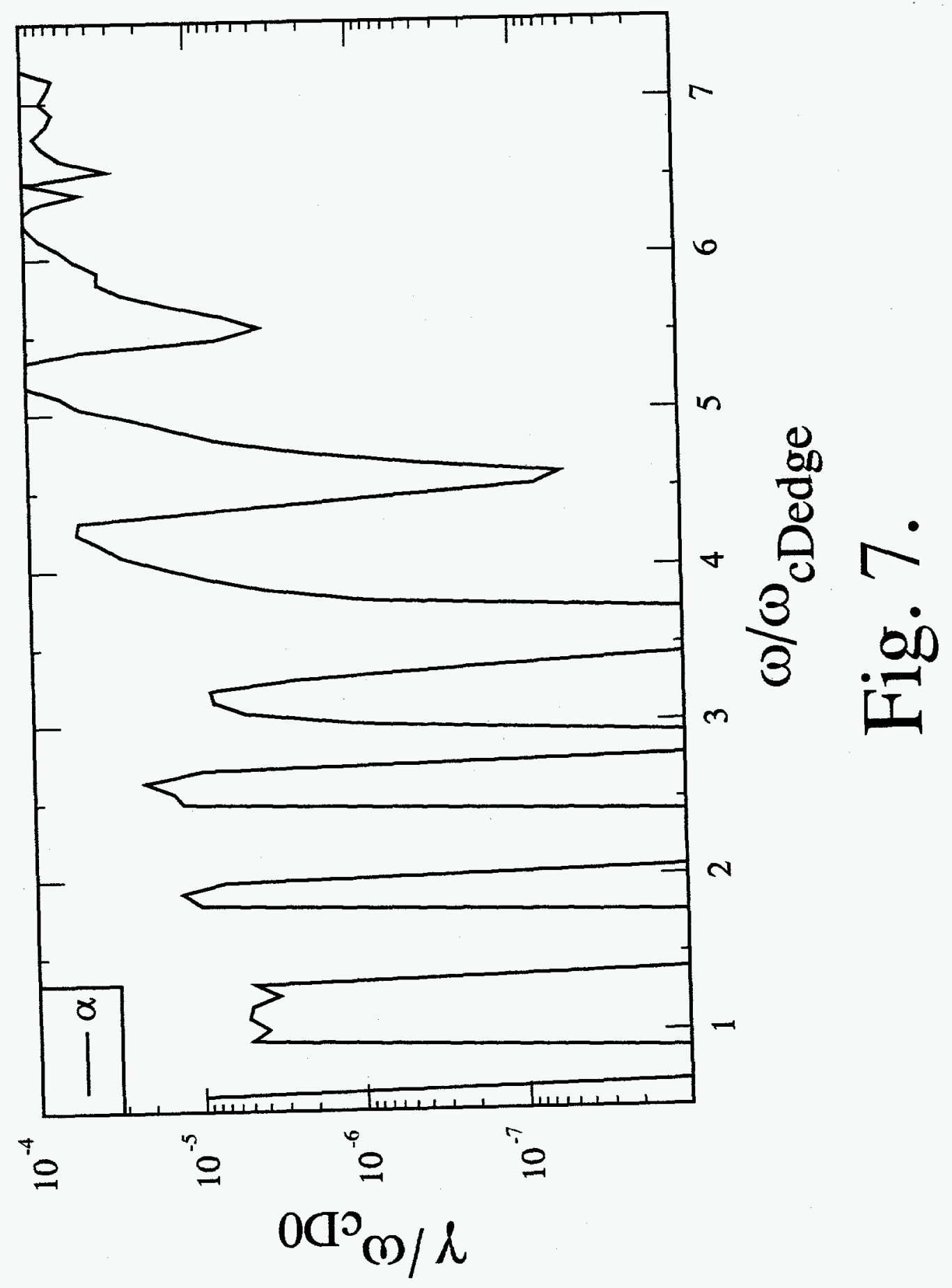


Dr. F. Paoloni, Univ. of Wollongong, AUSTRALIA

Prof. R.C. Cross, Univ. of Sydney, AUSTRALIA

Plasma Research Lab., Australian Nat. Univ., AUSTRALIA

Prof. I.R. Jones, Flinders Univ, AUSTRALIA

Prof. F. Cap, Inst. for Theoretical Physics, AUSTRIA

Prof. M. Heindler, Institut für Theoretische Physik, AUSTRIA

Prof. M. Goossens, Astronomisch Instituut, BELGIUM

Ecole Royale Militaire, Lab. de Phy. Plasmas, BELGIUM

Commission-European, DG. XII-Fusion Prog., BELGIUM

Prof. R. Bouciqué, Rijksuniversiteit Gent, BELGIUM

Dr. P.H. Sakanaka, Instituto Fisica, BRAZIL

Prof. Dr. I.C. Nascimento, Instituto Fisica, Sao Paulo, BRAZIL Instituto Nacional De Pesquisas Espaciais-INPE, BRAZIL Documents Office, Atomic Energy of Canada Ltd., CANADA Ms. M. Morin, CCFMTokamak de Varennes, CANADA Dr. M.P. Bachynski, MPB Technologies, Inc., CANADA Dr. H.M. Skarsgard, Univ. of Saskatchewan, CANADA Prof. J. Teichmann, Univ. of Montreal, CANADA Prof. S.R. Sreenivasan, Univ. of Calgary, CANADA Prof. R. Marchand, INRS-Energie et Materiaux, CANADA Dr. R. Bolton, Centre canadien de fusion magnétique, CANADA Dr. C.R. James, Univ. of Alberta, CANADA Dr. P. Lukác, Komenského Universzita, CZECHO-SLOVAKIA The Librarian, Culham Laboratory, ENGLAND Library, R61, Rutherford Appleton Laboratory, ENGLAND Mrs. S.A. Hutchinson, JET Library, ENGLAND Dr. S.C. Sharma, Univ. of South Pacific, FIJI ISLANDS

P. Mähönen, Univ. of Helsinki, FINLAND

Prof. M.N. Bussac, Ecole Polytechnique,, FRANCE

C. Mouttet, Lab. de Physique des Milieux lonisés, FRANCE

J. Radet, CENCADARACHE - Bat 506, FRANCE

Prof. E. Economou, Univ. of Crete, GREECE

Ms. C. Rinni, Univ. of loannina, GREECE

Preprint Library, Hungarian Academy of Sci., HUNGARY

Dr. B. DasGupta, Saha Inst. of Nuclear Physics, INDIA

Dr. P. Kaw, inst. for Plasma Research, INDIA

Dr. P. Rosenau, Israel Inst. of Technology, ISRAEL

Librarian, Intemational Center for Theo Physics, ITALY

Miss C. De Palo, Associazione EURATOM-ENEA, ITALY

Dr. G. Grosso, Istituto di Fisica del Plasma, ITALY

Prof. G. Rostangni, Istituto Gas lonizzati Del Cnr, ITALY
Dr. H. Yamato, Toshiba Res \& Devel Center, JAPAN

Prof. I. Kawakami, Hiroshima Univ., JAPAN

Prof. K. Nishikawa, Hiroshima Univ., JAPAN

Librarian, Naka Fusion Research Establishment, JAERI, JAPAN

Director, Japan Atomic Energy Research Inst., JAPAN

Prof. S. Itoh, Kyushu Univ., JAPAN

Research Info. Ctr., National Instit. for Fusion Scienć, JAPAN

Prof. S. Tanaka, Kyoto Univ., JAPAN

Library, Kyoto Univ., JAPAN

Prof. N. Inoue, Univ. of Tokyo, JAPAN

Secretary, Plasma Section, Electrotechnical Lab., JAPAN

Dr. O. Mitarai, Kumamoto Inst. of Technology, JAPAN

Dr. G.S. Lee, Korea Basic Sci. Ctr., KOREA

J. Hyeon-Sook, Korea Atomic Energy Research Inst., KOREA D.I. Choi, The Korea Adv. Inst. of Sai. \& Tech., KOREA Leandro Melendez Lugo, Inst. Nacy. de Inves. Nucl, MEXICO Prof. B.S. Liley, Univ. of Waikato, NEW ZEALAND Inst of Physics, Chinese Acad Sci PEOPLE'S REP. OF CHINA Library, Inst. of Plasma Physics, PEOPLE'S REP. OF CHINA Tsinghua Univ. Library, PEOPLE'S REPUBLIC OF CHINA Z. Li, S.W. Inst Physics, PEOPLE'S REPUBLIC OF CHINA Prof. J.A.C. Cabral, Instituto Superior Tecnico, PORTUGAL Prof. M.A. Hellberg, Univ. of Natal, S. AFRICA Prof. D.E. Kim, Pohang Inst. of Sci. \& Tech., SO. KOREA Prof. C.I.E.M.A.T, Fusion Division Library, SPAIN Dr. L Stenflo, Univ. of UMEA, SWEDEN Library, Royal Inst. of Technology, SWEDEN Prof. H. Wilhelmson, Chalmers Univ. of Tech., SWEDEN Centre Phys. Des Plasmas, Ecole Polytech, SWITZERLAND Bibliotheek, Inst. Voor Plasma-Fysica, THE NETHERLANDS Asst. Prof. Dr. S. Cakir, Middle East Tech. Univ., TURKEY Dr. V.A. Glukhikh,Sci. Res. Inst. Electrophys.I Apparatus, USSR Dr. D.D. Ryutov, Siberian Branch of Academy of Sci., USSR Dr. G.A. Eliseov, I.V. Kurchatov Inst., USSR Librarian, The Ukr.SSR Academy of Sciences, USSR Dr. LM. Kovrizhnykh, Inst. of General Physics, USSR Kemforschungsanlage GmbH, Zentralbibliothek, W. GERMANY Bibliothek, Inst. Für Plasmaforschung, W. GERMANY Prof. K. Schindler, Ruhr-Universitát Bochum, W. GERMANY Dr. F. Wagner, (ASDEX), Max-Planck-Institut, W. GERMANY Librarian, Max-Planck-Institut, W. GERMANY 